Article

\title{
Regular Solutions in Higher-Derivative Gravity ${ }^{\dagger}$
}

\author{
Breno L. Giacchini ${ }^{1, *(1)}$ and Tibério de Paula Netto ${ }^{2}$ \\ 1 Centro Brasileiro de Pesquisas Físicas, Rua Dr. Xavier Sigaud 150, Urca, Rio de Janeiro, RJ 22290-180, Brazil \\ 2 Departamento de Física, ICE, Universidade Federal de Juiz de Fora, Juiz de Fora, MG 36036-330, Brazil; \\ tiberiop@fisica.ufjf.br \\ * Correspondence: breno@cbpf.br \\ $\dagger$ Based on the talk given by B.L.G. at the Estate Quantistica Conference, Scalea, Italy, 11-15 June 2018.
}

Received: 31 October 2018; Accepted: 29 November 2018; Published: 2 December 2018

\begin{abstract}
Local gravitational theories with more than four derivatives can have remarkable quantum properties. Namely, they can be super-renormalizable and may be unitary in the Lee-Wick sense, if the massive poles of the propagator are complex. It is important, therefore, to also explore the classical aspects of these theories. In this talk we present recent results in this direction. Specifically, we discuss the effect that that higher-order terms can have on the Newtonian potential and related singularities.
\end{abstract}

Keywords: higher-derivative gravity; Lee-Wick gravity; non-local gravity; spacetime singularities

\section{Introduction}

Higher-derivative extensions of general relativity (GR) have, recently, been the object of intensive investigation. The main motivations for this come from the possibility of softening the tension between renormalizability and unitarity in the domain of perturbative quantum gravity. In fact, GR is not perturbatively renormalizable, and fourth-order gravity contains a ghost in the spectrum; nonetheless with six or more derivatives it is possible to restore the unitarity of the S-matrix if the ghost-like poles in the propagator are complex (Lee-Wick gravity) [1,2]. Alternatively, ghosts can be avoided if the original graviton propagator is modified by non-local factors which do not introduce any new pole in the complex plane [3-7]. In addition, the theory can be (super-)renormalizable if the propagator has an improved ultraviolet (UV) behavior $[4,8,9]$.

In the present work we review some results (see Refs. [10-14]) on a classical aspect of these higher-derivative gravity theories, namely the cancellation of the singularities in the linear regime. Our focus is on general polynomial-derivative theories, defined by the action [9]

$$
S_{\text {grav }}=\frac{1}{4 \kappa} \int d^{4} x \sqrt{-g}\left\{-2 R+R_{\mu \nu} F_{1}(\square) R^{\mu \nu}+R F_{2}(\square) R\right\},
$$

where $\kappa=8 \pi G$ and $F_{1}$ and $F_{2}$ are polynomial functions of the d'Alembertian, not necessarily of the same degree. In the linear regime this is the most general local action with higher derivatives, and it includes as particular case the Lee-Wick gravity.

Since the 1970s it is known that the case of trivial (constant non-null) polynomials yields a renormalizable theory with a finite modified Newtonian potential $[8,15]$. Nonetheless, curvature singularities are still present in both linear and non-linear regimes for static spherically symmetric solutions coupled to a point-like source [15-17]. In what follows we summarize recent generalizations of these results to the case of the polynomial-derivative theory (1) in the weak-field approximation. The main conclusion is that all the models with at least six derivatives in the scalar and the tensor sectors have not only a finite interparticle potential $[10,11]$ but also regular curvature invariants $[12,13]$. 


\section{Field Generated by a Static Point-Like Mass}

In the linear approximation one assumes the metric $g_{\mu v}=\eta_{\mu v}+h_{\mu \nu}$ to be a fluctuation around the Minkowski spacetime, and restricts considerations to the first order equations of motion for the field $h_{\mu \nu}$. For the action (1), supplemented by a matter action, the variational principle yields

$$
\begin{aligned}
& f_{2}(\square)\left(\square h_{\mu \nu}-\partial_{\rho} \partial_{\mu} h_{\nu}^{\rho}-\partial_{\rho} \partial_{\nu} h_{\mu}^{\rho}\right)+\frac{1}{3}\left[2 f_{0}(\square)+f_{2}(\square)\right]\left(\eta_{\mu v} \partial_{\rho} \partial_{\omega} h^{\rho \omega}-\eta_{\mu v} \square h+\partial_{\mu} \partial_{v} h\right) \\
& +\frac{2}{3}\left[f_{2}(\square)-f_{0}(\square)\right] \frac{1}{\square} \partial_{\mu} \partial_{\nu} \partial_{\rho} \partial_{\omega} h^{\rho \omega}=-2 \kappa T_{\mu v},
\end{aligned}
$$

where $T_{\mu \nu}$ is the energy-momentum tensor sourcing the field and the functions $f_{0}$ and $f_{2}$ are defined via

$$
\begin{aligned}
& f_{0}(z) \equiv 1+z F_{1}(z)+3 z F_{2}(z) \\
& f_{2}(z) \equiv 1-\frac{1}{2} z F_{1}(z) .
\end{aligned}
$$

For a static point-like source, i.e., $T_{\mu v}=\rho \delta_{\mu}^{0} \delta_{v}^{0}$ with $\rho(\mathbf{r})=M \delta(\mathbf{r})$, the metric perturbation can be written in isotropic coordinates as

$$
h_{\mu v}=\operatorname{diag}(-2 \Phi,-2 \Psi,-2 \Psi,-2 \Psi)
$$

Using the linearized equations of motion, it is possible to show that the metric potentials are given by [12]

$$
\Phi=\frac{1}{3}\left(2 \chi_{2}+\chi_{0}\right), \quad \Psi=\frac{1}{3}\left(\chi_{2}-\chi_{0}\right)
$$

where $\chi_{s}(s=0,2)$ satisfies

$$
f_{s}(\Delta) \Delta \chi_{s}=\kappa_{s} \rho
$$

with $\kappa_{0}=-\kappa / 2, \kappa_{2}=\kappa$.

As it was shown in [12], the solution for Equation (7) for a polynomial function $f_{s}(z)$ and a delta-source reads

$$
\chi_{s}(r)=-\frac{\kappa_{s} M}{4 \pi r}+\frac{\kappa_{s} M}{4 \pi^{3 / 2}} \sum_{i=1}^{N_{s}} \sum_{j=1}^{n_{(s) i}} \frac{a_{(s) i, j}}{(j-1) !}\left(\frac{r}{2 m_{(s) i}}\right)^{j-\frac{3}{2}} K_{j-\frac{3}{2}}\left(m_{(s) i} r\right),
$$

where $K_{v}$ is the modified Bessel function of the second kind. Also, $z=-m_{(s) i}^{2}$ is one of the $N_{s}$ distinct roots of the equation $f_{s}(-z)=0$, each of them with multiplicity $n_{(s) i}$. Of course, if $\mathcal{N}_{s}$ is the degree of $f_{s}(z)$, then $\sum_{i} n_{(s) i}=\mathcal{N}_{s}$. The coefficient $a_{(s) i, j}$ follows from the partial fraction decomposition of $\left[z f_{s}(-z)\right]^{-1}$ :

$$
-\frac{1}{z f_{s}(-z)}=-\frac{1}{z}+\sum_{i=1}^{N_{s}} \sum_{j=1}^{n_{(s) i}} \frac{a_{(s) i, j}}{\left(z+m_{(s) i}^{2}\right)^{j}} .
$$

We remark that these formulas hold also for the Lee-Wick gravity models, in which there are complex massive quantities $m_{(s) i}$. Moreover, they explicitly take into account the case of degenerate roots, which are possible only in polynomial-derivative theories. 


\section{Finiteness of the Metric Potentials}

From the comparison of both sides of Equation (9) one can show that $\sum_{i} a_{(s) i, 1}=1$ [12]. Since the expansion of Equation (8) around $r=0$ gives

$$
\chi_{s}(r)=-\frac{\kappa_{s} M}{4 \pi r}\left[1-\sum_{i=1}^{N_{s}} a_{(s) i, 1}\right]+\text { const. }+O(r)
$$

it follows that $\chi_{s}$ is finite provided that $N_{s} \geq 1$, i.e., if $f_{s}$ is a non-trivial polynomial. Therefore, the (modified) Newtonian metric potentials $\Phi$ and $\Psi$ are finite provided that the model contains at least four derivatives both in the spin- 2 and the spin- 0 sectors. This result was previously obtained for the case of super-renormalizable models with simple poles in [10]; and later is was extended to general polynomial models in [11], where the identity $\sum_{i} a_{(s) i, 1}=1$ was proved by means of a different approach.

\section{Curvature Regularity}

In isotropic spherical coordinates, the non-zero components of the Riemann curvature tensor associated with the metric perturbation (5) read

$$
\begin{aligned}
R_{t r t r} & =\Phi^{\prime \prime}, \\
R_{t \theta t \theta} & =r \Phi^{\prime}=\frac{R_{t \phi t \phi}}{\sin ^{2} \theta}, \\
R_{r \theta r \theta} & =r\left(\Psi^{\prime}+r \Psi^{\prime \prime}\right)=\frac{R_{r \phi r \phi}}{\sin ^{2} \theta}, \\
R_{\theta \phi \theta \phi} & =2 r^{3} \Psi^{\prime} \sin ^{2} \theta .
\end{aligned}
$$

In view of Equations (6) and (10) it follows that the finiteness of the potentials $\chi_{0}$ and $\chi_{2}$ imply in the finiteness of the Riemann tensor. This is not enough; however, to guarantee the absence of singularities in the curvature invariants-for instance, for the Kretschmann scalar one gets

$$
R_{\mu \nu \alpha \beta}^{2}=4\left(\Phi^{\prime \prime 2}+2 \Psi^{\prime \prime 2}\right)+\frac{16}{r} \Psi^{\prime} \Psi^{\prime \prime}+\frac{8}{r^{2}}\left(\Phi^{\prime 2}+3 \Psi^{\prime 2}\right)
$$

Hence, $\Phi^{\prime}(0)=\Psi^{\prime}(0)=0$ is a sufficient condition for having a regular Kretschmann invariant [18]. It turns out that this condition is a necessary one for the regularity of the whole set of curvature invariants [12]. In fact,

$$
\begin{aligned}
R= & 2\left(2 \Psi^{\prime \prime}-\Phi^{\prime \prime}\right)+\frac{4}{r}\left(2 \Psi^{\prime}-\Phi^{\prime}\right) \\
R_{\mu \nu}^{2}= & 2\left(\Phi^{\prime \prime 2}+3 \Psi^{\prime \prime 2}-2 \Phi^{\prime \prime} \Psi^{\prime \prime}\right)+\frac{4}{r}\left(\Phi^{\prime} \Phi^{\prime \prime}+5 \Psi^{\prime} \Psi^{\prime \prime}-\Psi^{\prime} \Phi^{\prime \prime}-\Phi^{\prime} \Psi^{\prime \prime}\right) \\
& +\frac{2}{r^{2}}\left(3 \Phi^{\prime 2}+11 \Psi^{\prime 2}-6 \Phi^{\prime} \Psi^{\prime}\right), \\
C_{\mu \nu \alpha \beta}^{2}= & \frac{4}{3 r^{2}}\left[r\left(\Phi^{\prime \prime}+\Psi^{\prime \prime}\right)-\left(\Phi^{\prime}+\Psi^{\prime}\right)\right]^{2} .
\end{aligned}
$$


It is straightforward to verify that the condition $\Phi^{\prime}(0)=\Psi^{\prime}(0)=0$ also regularizes the components of the Ricci and Weyl curvature tensors, whose non-zero components respectively read

$$
\begin{aligned}
& R_{t t}=\Phi^{\prime \prime}+2 \frac{\Phi^{\prime}}{r}, \\
& R_{r r}=2 \Psi^{\prime \prime}-\Phi^{\prime \prime}+2 \frac{\Psi^{\prime}}{r}, \\
& R_{\theta \theta}=r\left(3 \Psi^{\prime}-\Phi^{\prime}+r \Psi^{\prime \prime}\right)=\frac{R_{\phi \phi}}{\sin ^{2} \theta},
\end{aligned}
$$

and

$$
\begin{aligned}
C_{t r t r} & =\frac{1}{3}\left(\Phi^{\prime \prime}+\Psi^{\prime \prime}-\frac{\Phi^{\prime}+\Psi^{\prime}}{r}\right) \\
C_{t \theta t \theta} & =C_{r \theta r \theta}=\frac{C_{t \phi t \phi}}{\sin ^{2} \theta}=\frac{C_{r \phi r \phi}}{\sin ^{2} \theta}=-\frac{1}{2} r^{2} C_{t r t r}, \\
C_{\theta \phi \theta \phi} & =-r^{4} \sin ^{2} \theta C_{t r t r} .
\end{aligned}
$$

Proceeding the expansion (10) of the auxiliary potentials $\chi_{s}$ around $r=0$ up to the linear term one can show that [12]

$$
\chi_{s}^{\prime}(0)=\frac{\kappa_{s} M}{8 \pi}\left(\sum_{i=1}^{N_{s}} a_{(s) i, 1} m_{(s) i}^{2}-\sum_{i=1}^{N_{s}} a_{(s) i, 2}\right) .
$$

Moreover, from the definition of the coefficients $a_{(s) i, j}$ through the partial fraction decomposition (9) one can prove that the quantity between parenthesis in Equation (25) above is identically null if $\mathcal{N}_{s}>1$ (i.e., if the degree of $f_{s}(z)$ is at least two), and it is non-zero if $\mathcal{N}_{s}=1[12,13]$. Therefore, it follows that the necessary and sufficient condition for having regular curvature invariants in polynomial-derivative gravity is to have at least six derivatives both in the spin- 2 and spin- 0 sectors.

In this sense, fourth-derivative gravity would be the only local higher-derivative model which always have curvature singularities when coupled to a delta-source. In higher-order theories this can only happen for specific choices of parameters, namely, if $F_{2}=$ const. or $F_{2}=-3 F_{1}$, see Equations (3) and (4).

\section{Higher Derivatives as Source Regularization}

The regularization of the Newtonian singularities as one goes from 2 to 4 (potential singularity) and then to six and more derivatives (curvature singularities) can be viewed as a regularization of the effective source $\varrho_{s}$ defined through

$$
\rho(r)=f_{s}(\Delta) \varrho_{s}(r)
$$

With this definition, (7) can be cast as a Poisson equation with the new source,

$$
\Delta \chi_{s}=\kappa_{s} \varrho_{s} .
$$

It is possible to show that if $f_{s}(z)$ is a linear polynomial then $\varrho_{s}$ gets smeared but it still blows up in the origin. Nonetheless, if $f_{s}(z)$ is at least of quadratic order, then the corresponding effective source is regular, which regularizes the potential $\chi_{s}$ (i.e., it ensures that $\left.\chi_{s}^{\prime}(0)=0\right)$ [13]. In this spirit, the complete regularization of the delta-source coincides with the avoidance of curvature singularities. Qualitatively, the mechanisms by which the higher derivatives allow to eliminate the singularities are the smearing and regularization of the effective delta-source. Ultimately, this is achieved by the improved behavior of the propagator in the UV [13]. 
Working with effective sources is useful for extending considerations to non-local ghost-free models, in which the functions $F_{1}$ and $F_{2}$ in the action are non-polynomial ones. Indeed, in [13] it was shown that the regularity of weak field limit solutions of the polynomial gravity implies in the regularity of analogous solutions in a large class of non-local models (see, e.g., [18-23]). Nonetheless, non-locality and/or ghost-free conditions do not ensure that the model is free from curvature singularities, one example of this kind was presented in [13].

\section{Conclusions}

In the present work we reviewed recent results concerning the occurrence of regular solutions in polynomial-derivative gravity. It is remarkable that, contrary to what one might expect considering only the fourth-order gravity, the asymptotically flat, static spherically symmetric solutions in linearized higher-derivative gravity are, in general, singularity-free. This fact is closely related to the improved UV behavior of the propagator [13].

A natural question is whether the regularity of the solutions is preserved in the full non-linear regime. In the case of fourth-order gravity it has been shown that the singularity is present in both regimes [15-17]. On the other hand, the numerical search for static spherically symmetric solutions in models with 6, 8 and 10 derivatives presented in [24] only found regular solutions. This subject certainly deserves more investigation, also in the case of non-local gravities.

Finally, we mention that the regularity of the metric for collapsing spherical null shells in general polynomial models was analyzed in [12], generalizing previous considerations of [18]. The results are analogous to the Newtonian case. For thick null shells the curvature singularity is present only for the theory with four derivatives, while the models with six or more derivatives have regular curvature invariants. Other phenomenological applications of polynomial gravity can be found in, e.g., Refs. [14,25].

Author Contributions: The authors contributed equally to this work.

Funding: This research was partially funded by CNPq, grant number 140371/2015-0, and by CAPES, through the PNPD program.

Acknowledgments: Some results mentioned in this review were obtained in original works that were done in collaboration with A. Accioly, L. Modesto and I.L. Shapiro. We gratefully acknowledge these collaborations.

Conflicts of Interest: The authors declare no conflict of interest.

\section{References}

1. Modesto, L.; Shapiro, I.L. Superrenormalizable quantum gravity with complex ghosts. Phys. Lett. B 2016, 755, 279-284. [CrossRef]

2. Modesto, L. Super-renormalizable or finite Lee-Wick quantum gravity. Nucl. Phys. B 2016, 909, 584-606. [CrossRef]

3. Krasnikov, N.V. Nonlocal Gauge Theories. Theor. Math. Phys. 1987, 73, 1184-1190 [CrossRef]

4. Kuzmin, Y.V. The Convergent Nonlocal Gravitation. Sov. J. Nucl. Phys. 1989, 50, 1011-1014.

5. Tomboulis, E.T. Superrenormalizable gauge and gravitational theories. arXiv 1997, arXiv:hep-th/9702146.

6. Modesto, L. Super-renormalizable quantum gravity. Phys. Rev. D 2012, 86, 044005. [CrossRef]

7. Biswas, T.; Gerwick, E.; Koivisto, T.; Mazumdar, A. Towards Singularity- and Ghost-Free Theories of Gravity. Phys. Rev. Lett. 2012, 108, 031101. [CrossRef] [PubMed]

8. Stelle, K.S. Renormalization of Higher Derivative Quantum Gravity. Phys. Rev. D 1977, 16, 953-969. [CrossRef]

9. Asorey, M.; Lopez, J.L.; Shapiro, I.L. Some remarks on high derivative quantum gravity. Int. J. Mod. Phys. A 1997, 12, 5711-5734. [CrossRef]

10. Modesto, L.; Netto, T.D.; Shapiro, I.L. On Newtonian singularities in higher derivative gravity models. J. High Energy Phys. 2015, 2015, 98, arXiv:1412.0740.

11. Giacchini, B.L. On the cancellation of Newtonian singularities in higher-derivative gravity. Phys. Lett. B 2017, 766, 306-311. [CrossRef] 
12. Giacchini, B.L.; Netto, T.D. Weak-field limit and regular solutions in polynomial higher-derivative gravities. arXiv 2018, arXiv:1806.05664.

13. Giacchini, B.L.; Netto, T.D. Effective delta sources and regularity in higher-derivative and ghost-free gravity. arXiv 2018, arXiv:1809.05907.

14. Accioly, A.; Giacchini, B.L.; Shapiro, I.L. Low-energy effects in a higher-derivative gravity model with real and complex massive poles. Phys. Rev. D 2017, 96, 104004. [CrossRef]

15. Stelle, K.S. Classical Gravity with Higher Derivatives. Gen. Relativ. Gravit. 1978, 9, 353-371. [CrossRef]

16. Lü, H.; Perkins, A.; Pope, C.N.; Stelle, K.S. Black Holes in Higher-Derivative Gravity. Phys. Rev. Lett. 2015, 114, 171601. [CrossRef]

17. Lü, H.; Perkins, A.; Pope, C.N.; Stelle, K.S. Spherically Symmetric Solutions in Higher-Derivative Gravity. Phys. Rev. D 2015, 92, 124019. [CrossRef]

18. Frolov, V.P. Mass-gap for black hole formation in higher derivative and ghost free gravity. Phys. Rev. Lett. 2015, 115, 051102. [CrossRef] [PubMed]

19. Frolov, V.P.; Zelnikov, A.; Netto, T.D. Spherical collapse of small masses in the ghost-free gravity. J. High Energy Phys. 2015, 2015, 107, arXiv:1504.00412.

20. Frolov, V.P.; Zelnikov, A. Head-on collision of ultrarelativistic particles in ghost-free theories of gravity. Phys. Rev. D 2016, 93, 064048. [CrossRef]

21. Edholm, J.; Koshelev, A.S.; Mazumdar, A. Behavior of the Newtonian potential for ghost-free gravity and singularity-free gravity. Phys. Rev. D 2016, 94, 104033. [CrossRef]

22. Buoninfante, L.; Koshelev, A.S.; Lambiase, G.; Mazumdar, A. Classical properties of non-local, ghost- and singularity-free gravity. J. Cosmol. Astropart. Phys. 2018, 1809, 034, arXiv:1802.00399.

23. Buoninfante, L.; Koshelev, A.S.; Lambiase, G.; Marto, J.; Mazumdar, A. Conformally-flat, non-singular static metric in infinite derivative gravity. J. Cosmol. Astropart. Phys. 2018, 2018, 014, arXiv:1804.08195.

24. Holdom, B. On the fate of singularities and horizons in higher derivative gravity. Phys. Rev. D 2002, 66, 084010. [CrossRef]

25. Accioly, A.; Giacchini, B.L.; Shapiro, I.L. On the gravitational seesaw in higher-derivative gravity. Eur. Phys. J. C 2017, 77, 540, arXiv:1604.07348.

(C) 2018 by the authors. Licensee MDPI, Basel, Switzerland. This article is an open access article distributed under the terms and conditions of the Creative Commons Attribution (CC BY) license (http://creativecommons.org/licenses/by/4.0/). 